\title{
MR-augmented cardio pulmonary exercise testing: an integrated approach to assessment of children with pulmonary hypertension
}

\author{
Nathaniel J Barber ${ }^{*}$, Vivek Muthurangu \\ From 19th Annual SCMR Scientific Sessions \\ Los Angeles, CA, USA. 27-30 January 2016
}

\section{Background}

Pulmonary arterial hypertension (PAH) is a life limiting disorder resulting in progressive right heart (RV) failure. Cardiac MRI is the reference standard for RV assessment in $\mathrm{PH}$ and has known prognostic value in children. Symptoms of PAH are exacerbated or become apparent with exercise and cardiopulmonary exercise testing (CPET) is also prognostic in PAH.

We have recently demonstrated the feasibility of MR augmented CPET (MR-CPET) in healthy adults. Using the Fick principle MR-CPET allows calculation of tissue oxygen extraction $\Delta \mathrm{cO}_{2}$. A combined approach using MR-CPET could provide a more sensitive method of assessing disease severity, the causes of exercise limitation and response to treatment. In this study we assessed the utility of MR-CPET in children with PAH.

\section{Methods}

10 Children with PAH aged 8 to 16 years (WHO class I or II, on oral/inhaled therapy only) and 10 healthy volunteers matched for age and sex underwent MRCPET. Exercise was performed on a MRI compatible ergometer (Lode, Groningen, The Netherlands) CPET data was collected using a commercial respiratory gasanalyser (Medgraphics, St. Paul, USA) with a modified MR compatible sampling umbilical.

Resting and peak exercise ventricular volumes and septal curvature were measured using a radial k-t SENSE real time sequence. Aortic flow was simultaneously continuously measured using a previously validated real-time UNFOLD-SENSE spiral PCMR sequence.

MR data was used to derive CO, HR and SV curves during exercise. Arteriovenous oxygen content gradient

Institute of Cardiovascular Science, UCL, London, United Kingdom
(AVO2) curves (a measure of tissue oxygen extraction) were calculated by dividing the $\mathrm{VO} 2$ and $\mathrm{CO}$ curves (Figure 1). Resting and peak exercise mean pulmonary
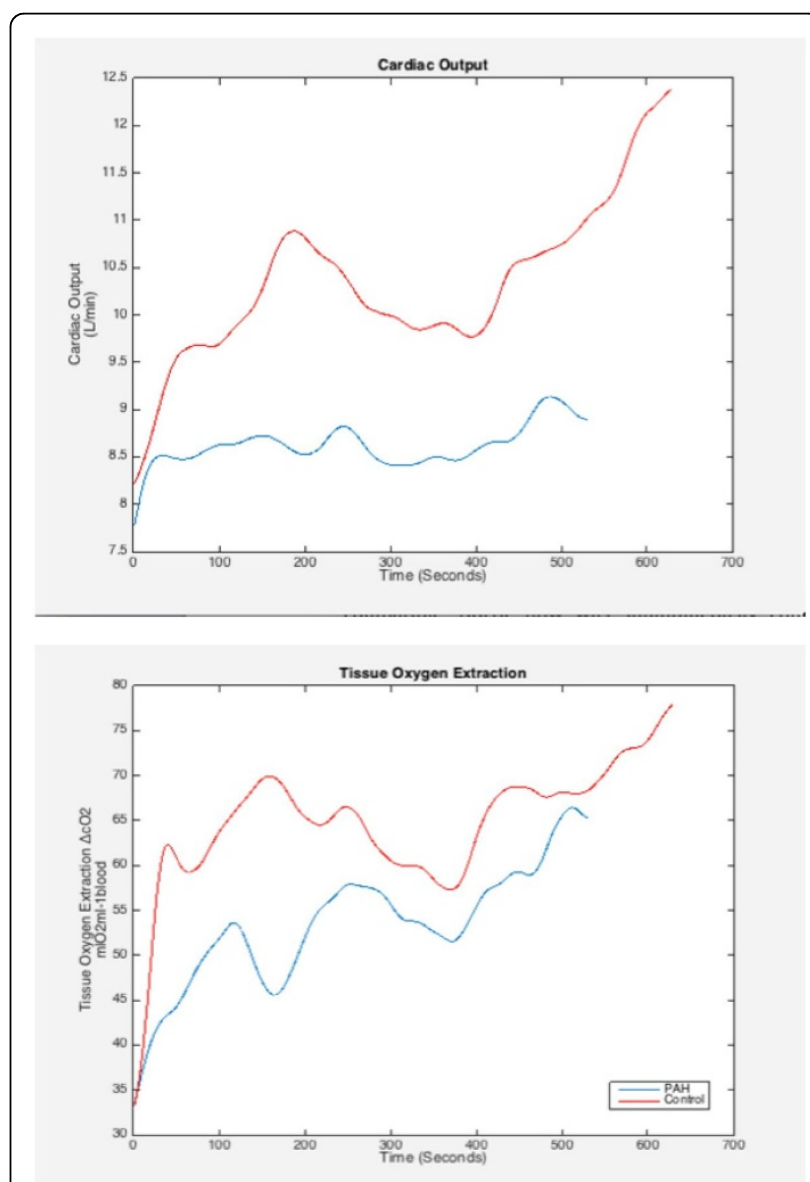

Figure 1 a) Cardiac output b) Oxygen extraction. 
artery pressures (mPAP) were estimated based on septal curvature using a previously validated technique.

Subjects also completed a six-minute walk test, experience survey and exercise history.

\section{Results}

All children successfully completed the test without complication.Peak $\mathrm{VO}_{2}$ was significantly lower in the PAH group. This seems to be driven by both reduced tissue oxygen extraction and cardiac output although only reduced $\Delta \mathrm{cO}_{2}$ was of statistical significance. MR-CPET measures at rest and exercise are shown in Figure 2.

RVEF was significantly lower in the PAH group both at rest and at peak exercise.There was a significant difference in the increase in estimated MPAP between the control and PAH groups. There was no significant

\begin{tabular}{|c|c|c|c|}
\hline Variable & Control group & $\mathrm{PAH}$ group & p Value \\
\hline $\begin{array}{l}\text { Resting } \mathrm{VO}_{2} \\
\left(\mathrm{ml}^{-} / \mathrm{min}^{-1}\right)\end{array}$ & $191(157-232)$ & $181(161-204)$ & 0.6182 \\
\hline $\begin{array}{l}\text { Peak VO } \\
\left(\mathrm{ml}_{2} / \mathrm{min}^{-1}\right)\end{array}$ & 803 (676-931) & $578(463-694)$ & .00834 \\
\hline $\begin{array}{l}\text { Resting cardiac } \\
\text { output, } \mathrm{Lmin}^{-1}\end{array}$ & $6.5(5-\quad 8)$ & $6.1(5.3-7)$ & .6359 \\
\hline $\begin{array}{l}\text { Peak cardiac output, } \\
\text { Lmin }^{-1}\end{array}$ & $9.8(8-12)$ & $8.1(7-9.2)$ & .0831 \\
\hline $\begin{array}{c}\text { Resting stroke } \\
\text { volume, } \mathrm{ml} / \text { beat }\end{array}$ & $71(56-85)$ & $66(57-76)$ & .5742 \\
\hline $\begin{array}{c}\text { Peak Stroke volume, } \\
\mathrm{ml} / \text { beat }\end{array}$ & $67(54-80)$ & $62(51-72)$ & .502 \\
\hline $\begin{array}{l}\text { Resting heart rate, } \\
\text { bpm }\end{array}$ & $83(71-95)$ & $81(74-88)$ & .7924 \\
\hline $\begin{array}{c}\text { Peak heart rate, } \\
\text { bpm }\end{array}$ & $149(136-161)$ & $133(121-145)$ & .06275 \\
\hline $\begin{array}{l}\text { Resting tissue } \\
\text { extraction } \Delta \mathrm{cO}_{2} \text {, } \\
\mathrm{mlO}_{2} \mathrm{ml}^{-1} \text { blood }\end{array}$ & $191(157-232)$ & $181(61-204)$ & 0.6182 \\
\hline $\begin{array}{c}\text { Peak tissue } \\
\text { extraction } \Delta \mathrm{cO}_{2} \text {, } \\
\mathrm{mlO}_{2} \mathrm{ml}^{-1} \mathrm{blood}\end{array}$ & $803(676-931)$ & $578(463-694)$ & .00834 \\
\hline $\mathrm{VE} / \mathrm{VCO}_{2}$ & $38(35-42)$ & $43(39-47)$ & .04454 \\
\hline $\begin{array}{c}\text { Estimated mPAP } \\
\text { rest, } \\
\mathrm{mmHg} \\
\end{array}$ & $11(9-14)$ & $32(25-41)$ & 0.00 \\
\hline $\begin{array}{c}\text { Estimated mPAP } \\
\text { exercise, } \\
\mathrm{mmHg}\end{array}$ & $15(12-17)$ & $58(47-71)$ & 0.00 \\
\hline $\begin{array}{c}\text { Difference mPAP, } \\
\text { rest - exercise. } \\
\mathrm{mmHg}\end{array}$ & $3.7(2.2-6.1)$ & $20(12-35)$ & 0.0001 \\
\hline RVEF rest & $62(58-65)$ & $56(51-60)$ & .03002 \\
\hline RVEF peak & $69(63-75)$ & $60(55-65)$ & .01855 \\
\hline $\begin{array}{l}\text { Six minute walk test } \\
\text { distance, } m\end{array}$ & 452 (418- 489) & $451(400-507)$ & 0.9578 \\
\hline
\end{tabular}


difference in 6-minute walk test distance between the groups.

\section{Conclusions}

MR-CPET is well tolerated in both healthy children and children with class I/II PAH. This study demonstrates that exercise intolerance in children with $\mathrm{PAH}$ is in part due to reduced tissue oxygen extraction and may open possibilities to study new pharmacological or exercise based therapeutic strategies.

Published: 27 January 2016

doi:10.1186/1532-429X-18-S1-P178

Cite this article as: Barber and Muthurangu: MR-augmented cardio pulmonary exercise testing: an integrated approach to assessment of children with pulmonary hypertension. Journal of Cardiovascular Magnetic Resonance 2016 18(Suppl 1):P178.

Submit your next manuscript to BioMed Central and take full advantage of:

- Convenient online submission

- Thorough peer review

- No space constraints or color figure charges

- Immediate publication on acceptance

- Inclusion in PubMed, CAS, Scopus and Google Scholar

- Research which is freely available for redistribution

Submit your manuscript at www.biomedcentral.com/submit 